\title{
Resistencia al desgaste de materiales de las orugas de tractores después del proceso de recuperación
}

Cristovão A. Ferreira Castro $^{1}$, Juan Ignacio Pereira ${ }^{2}$, Ossimar Maranho ${ }^{2}$, Carlos Henrique da Silva ${ }^{2}$.

\author{
${ }^{1}$ Instituto Federal de Educação, Ciência e Tecnologia do Amazonas - IFAM, Avenida Sete de Setembro, 1975, CEP: \\ 69020-120, Manaus, AM, Brasil. e-mail: cristovao@ifam.edu.br \\ ${ }^{2}$ Laboratório de Superfícies e Contato - LASC, Universidade Tecnológica Federal do Paraná -PPGEM/UTFPR, \\ Avenida Sete de Setembro, 3165, CEP: 80230-901, Curitiba, PR, Brasil. \\ e-mail: jipagudelo@hotmail.com ,maranho@utfpr.edu.br, carloshs@utfpr.edu.br
}

\begin{abstract}
RESUMO
Este estudio propone una alternativa para la recuperación de las orugas de tractores Bulldozer desgastadas por el efecto abrasivo del suelo. Para este fin, se aborda un análisis comparativo entre la resistencia al desgaste de una oruga original con dos orugas semejantes, recuperadas por procesos distintos. El primer proceso de recuperación utiliza la aplicación de un postizo en la región de desgaste y el segundo consiste de la recuperación de la región afectada mediante deposición por soldadura de un revestimiento duro. Las probetas fueron sometidas a ensayos de desgaste tipo rueda de caucho y chorro de arena seca (ASTM G 65-04); el análisis microestructural fue realizado por medio de la utilización de las técnicas de análisis de microscopia óptica (MO) y microscopia electrónica de barrido (SEM). Se obtiene como micromecanismo de desgaste predominante en la soldadura el microcorte, en tanto que para los demás materiales (ambos aceros) se observa deformación plástica y presencia de microsurcos. Fue posible observar que el proceso de recuperación de orugas utilizando el revestimiento con electrodo E-83.58 mostro ser una alternativa para este tipo de recuperación.
\end{abstract}

Palavras-chave: Revestimiento duro, Desgaste abrasivo, Soldadura, Electrodo revestido, Oruga.

\begin{abstract}
This study proposes an alternative for the recovery of the crawler tractor bulldozer worn by the abrasive effect of the soil. To this end, deals with a comparative analysis between the wear resistance of an original track like two caterpillars, recovered by different processes. The first recovery process uses the application of a hairpiece in the region of wear and the second is the recovery of the region affected by welding deposition of a hard coating. The specimens were subjected to wear tests type rubber wheel (ASTM G 65-04), the microstructural analysis was performed through the use of techniques for analysis of optical microscopy (OM) and scanning electron microscopy (SEM). The microcut was the wear micromechanism predominant in welding deposition. While for other materials (with lower hardness and less wear resistance) shows the presence of plastic deformation. Was observed that the recovery process tracked using the electrode coating E-83.58 showed to be an alternative for this type of recovery.
\end{abstract}

Keywords: Hard Coating, Abrasive wear, Welding, Coated Electrode, Crawler.

\section{INTRODUCCIÓN}

En la industria mundial, una de las mayores preocupaciones es el desgaste de los equipos, debido a que es la más frecuente causa de falla de los equipos u parte de ellos, ocasionando pérdidas por mantenimiento o reposición [1].

Es importante tener el conocimiento de las características físicas y las condiciones ambientales presentes en la interacción de las superficies de pares mecánicos que están en contacto, conociéndose el conjunto total como tribosistema estando compuesto por cuerpo y contracuerpo, elemento intermedio, ambiente (gases y/o líquidos, en diferentes condiciones de presión)[2]]. 
El desgaste abrasivo es definido como el desplazamiento de material causado por la presencia de partículas duras (o protuberancias), entre o incorporadas, en una o ambas superficies en movimiento [3]].

El contacto entre abrasivos y los cuerpos del sistema es clasificado tradicionalmente en dos tipos: abrasión a dos cuerpos y a tres cuerpos. En la situación en la cual la velocidad a la que el abrasivo golpea la superficie desgastada es una variable importante, el desgaste es clasificado como abrasión por erosión [4]. La interpretación usual define a la abrasión a dos cuerpos como el sistema en el cual las partículas o las asperezas están rígidamente fijas en el segundo cuerpo, haciendo con que penetren y causen cortes en el primer cuerpo. Por otro lado, la abrasión a tres cuerpos los abrasivos están libres para desplazarse. Los ensayos de desgaste abrasivo a dos cuerpos producen tasas de desgaste de por lo menos un orden de grandeza mayores que la abrasión a tres cuerpos, cuando están sobre condiciones comparables []ㅡ.

GATES [6] discute dos ejemplos de conflictos existentes con el uso de tal clasificación. El ensayo de rueda de caucho y chorro de arena [7], el cual es usualmente clasificado como un sistema a tres cuerpos puede adquirir la configuración a dos cuerpos, debido a la posibilidad de que los abrasivos queden incrustados en el caucho. Dependiendo de las características del material del contracuerpo utilizado en los ensayos a tres cuerpos, como la dureza y la tenacidad, las partículas abrasivas pueden desgastar la superficie de este, ocasionando apenas mecanismo de corte, o rueden ocurriendo apenas impresiones.

Este equipo permite realizar ensayos a seco o lubricado, con alta confiabilidad de resultados, siendo empleado tradicionalmente en la industria minera. Es muy común la implementación de modificaciones específicas en la norma ASTM G 65-04 [7] dependiendo de las condiciones de trabajo y de los mecanismos de desgaste que se desean estudiar.

Estas modificaciones están relacionadas con el tipo de material abrasivo, tipos de muestras (cuerpo y contracuerpo), además de parámetros de ensayo (velocidad e carga).

HUTCHINGS [] ] modificó el sistema de alimentación de partículas abrasivas del ensayo para garantizar la formación de una capa de material abrasivo en la superficie de la probeta y obtener el desgaste de manera más homogénea. NAHVI [9] propuso modificaciones que consisten en variaciones en el tamaño de la naturaleza de las partículas abrasivas. PEREIRA [10] utilizo diferentes morfologías del material abrasivo además de modificar la naturaleza del mismo, en el estudio de desgaste de materiales utilizados en la industria de moldes para inyección de plásticos.

RUTHERFORD y HUTCHINGS [11] mostraron que en el desgaste abrasivo a tres cuerpos ocurre una rotación de partículas, resultando en impresiones ubicadas a lo largo de la superficie de desgaste. Cortes/surcos están asociados a la adhesión de las partículas en el contracuerpo, que deslizan sobre la muestra.

Los efectos del tamaño de las partículas abrasivas sobre la resistencia al desgaste han sido intensamente referenciados en la bibliografía; FANG et al. [12] destacan la importancia de la morfología del abrasivo en la determinación del mecanismo de desgaste en sistemas lubricados. Estos autores concluyeron que las partículas con un formato más cercano a la esfera tienen la tendencia de desgastar en una de las superficies y deslizarse sobre la otra, mientras que partículas con un formato que presente picos tienden a rodar presentando marcas de impresión en las superficies.

Los mecanismos de desgaste abrasivo pueden ser identificados en dos tipos: Los que son controlados por deformación plástica y aquellos controlados por fractura frágil. El micro-corte es el mecanismo controlado por deformación plástica más efectivo para la remoción de material. En este mecanismo, el abrasivo, actúa como herramienta de corte, y el material removido se presenta en forma de astillas $[\underline{13}]$.

La asociación entre micromecanismos y sus tasas de desgaste, conforme varia la razón $\mathrm{H}_{\mathrm{a}} / \mathrm{H}$ (dureza del abrasivo por la dureza del material desgastado) fue presentada por KHRUSCHOV [14].

Según el investigador, los mecanismos asociados a la fatiga de bajo ciclo, como las 'microimpresiones', están relacionadas con bajas razones $\mathrm{H}_{\mathrm{a}} / \mathrm{H}$, caracterizando el régimen de desgaste como moderado, mientras que el mecanismo de microcorte estaría asociado con el régimen severo e con altas razones $\mathrm{H}_{\mathrm{a}} / \mathrm{H}$. Usualmente se utiliza la razón $\mathrm{H}_{\mathrm{a}} / \mathrm{H}$ para la previsión de los regímenes de desgaste apenas para las situaciones en las cuales son verificados mecanismos controlados por deformación plástica.

La lucha contra el desgaste de las áreas de mantenimiento de las industrias es incesante, intentando aumentar el tiempo entre paradas de mantenimiento o reducir el cambio de los componentes. Según MONTEIRO y CUNHA [15] la posibilidad de reconstruir estos componentes o prepararlos para soportar 
las condiciones de trabajo en aplicaciones que exigen grandes respuestas mecánicas o fenómenos complejos de desgaste, está constantemente estudiada y nuevas técnicas son propuestas siempre en el sentido de aumentar la vida útil y reducir los tiempos y costos. Una de las técnicas más utilizadas es la aplicación de revestimientos que reducen el desgaste, siendo este hecho mediante la utilización de técnicas de soldadura entre otros procesos disponibles, con excelentes resultados, en muchos casos alcanzando tiempos de vida superiores a las piezas nuevas.

La soldadura de revestimientos está asumiendo importancia cada vez mayor para realizar reconstrucciones de piezas deterioradas además de la deposición de camadas protectoras con bajo costo operacional, reduciendo el tiempo de recuperación de las piezas y el tiempo de la producción, convirtiéndose en una solución práctica, viable y de técnica sencilla [16]. Según BUCHELY et al. [17], aplicar un material de elevada dureza es una de las formas más útiles y económicas de mejorar el desempeño de los componentes sometidos a condiciones severas de desgaste. FERNANDES et al. [18] relatan que los componentes de máquinas de la industria minera se ven frecuentemente envueltos en procesos de desgaste severo. Este es el caso de los apoyos de las ruedas de las excavadoras en mina a cielo abierto.

Las excavadoras, motivo de este estudio (Figura 1a) se diferencia de los demás tipos de tractores por el tipo de tren de desplazamiento, está compuesto de dos orugas formadas por la unión de varias orugas. Las orugas, Figura 1b del tren de movimiento, tienen dos funciones, la primera soportar el peso de la máquina y la segunda proporcionar la tracción a través del atrito con el suelo para permitir que el tractor ejecute su función de remoción de material. Para RICARDO y CATALANI [19] los tractores de orugas (Bulldozer) presentan elevado esfuerzo de tracción, conjugado con una buena adherencia sobre el terreno permitiéndoles retirar o empujar grandes cargas sin tener el problema de deslizamiento aún en lugares de desnivel grande.

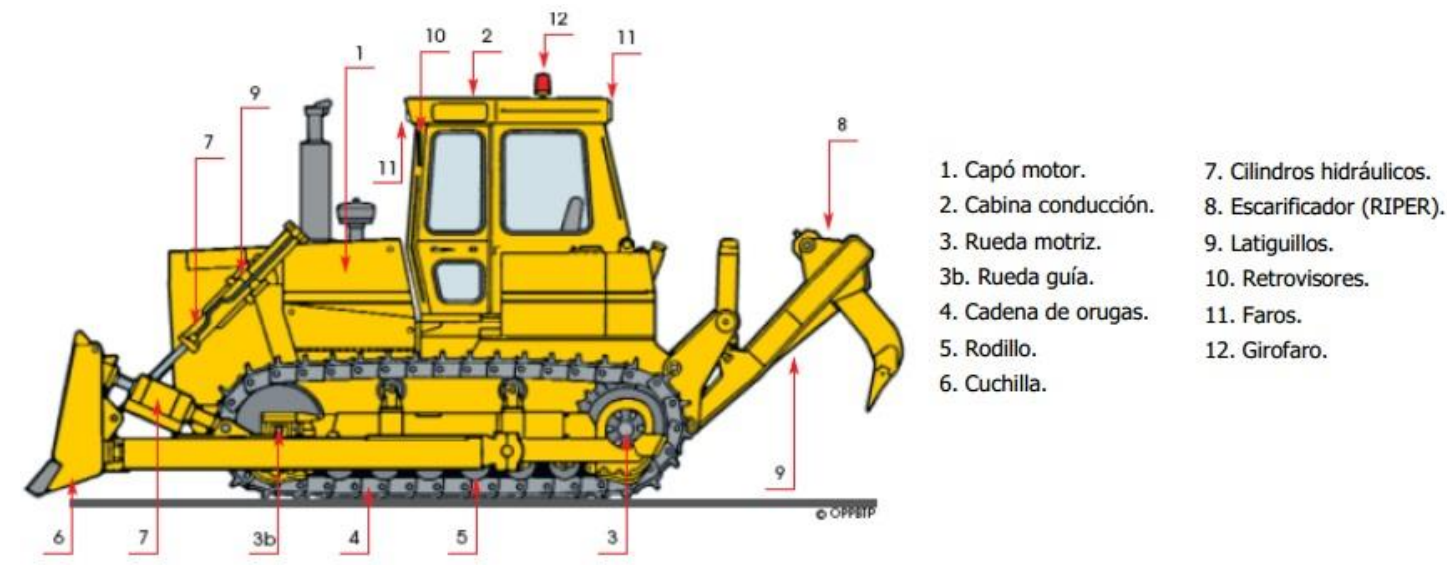

(a)

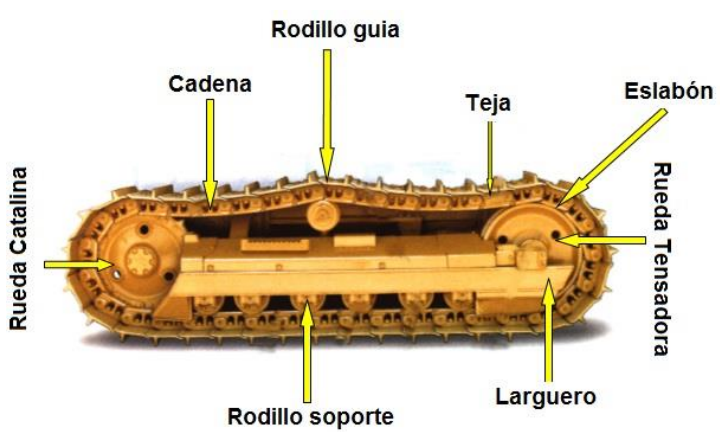

(b)

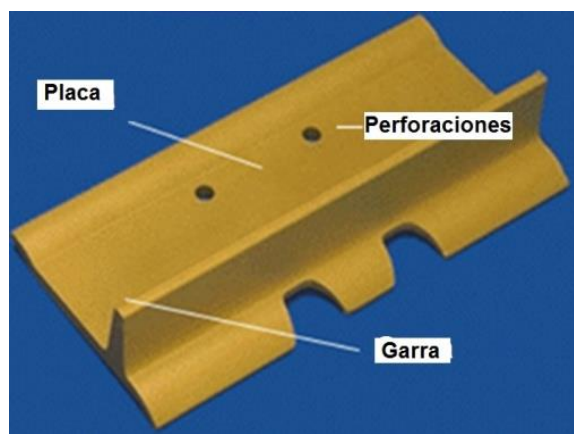

(c)

Figura 1: Excavadoras tipo Bulldozer: (a) partes principales de un tractor de orugas, (b) detalle del tren de movimiento del tractor, (c) placa de la oruga del tractor [20].

Básicamente tres métodos son utilizados en la recuperación de las orugas; cambio de la pieza completa por una semejante a la original, la adición de un postizo utilizando soldadura en la región desgastada o la aplicación de un revestimiento duro utilizando soldadura de arco eléctrico como es 
presentado en la Figura 2.

En este trabajo los ensayos fueron todos de tipo abrasivo, simulando lo que se obtiene en la vida común, con el objetivo de evaluar el comportamiento de cada uno de los métodos de recuperación de las piezas y analizar cual presenta mejor resultado a la hora de elegir uno de los métodos para utilización en el campo.

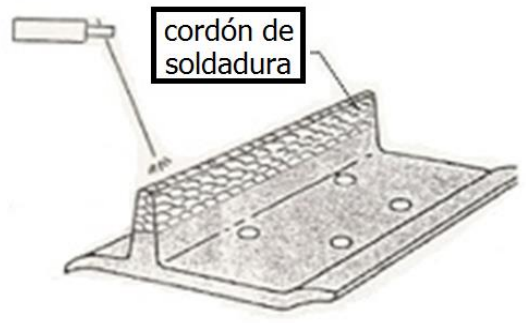

(a)

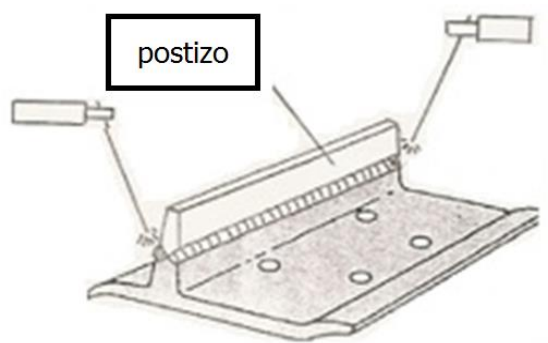

(b)

Figura 2: Ejemplos de recuperación de las orugas: (a) cordón de soldadura y (b) adición de postizo (adaptado de SOTREQ [21]).

\section{PROCEDIMIENTO EXPERIMENTAL}

\subsection{Materiales}

Para los ensayos fueron fabricados nueve probetas prismáticas con dimensiones 76,2 x 25,4 x 12,7 mm, en grupos de tres como son presentados a continuación:

a) Oruga original: Fue utilizada la oruga original de un tractor Bulldozer fabricada en acero AISI 5140, laminada y templada, donada por una empresa encargada de trabajo en vías. La composición química es presentada en la Tabla 1. Las probetas fueron elaboradas mediante utilización de un centro de maquinado y fueron extraídos de la región central de la garra de una oruga original;

b) Postizo: El postizo utilizado para la fabricación de las probetas fue retirado de una oruga recuperada por la adición de un postizo. Por lo tanto se trata de una pieza que recibió una carga térmica proveniente del proceso de soldadura;

c) Cordón de soldadura: Las probetas fabricadas utilizando soldadura con electrodo revestido SMAW (Shielded Metal Arc Welding) utilizando parte de una oruga nueva como sustrato y con la adición de un consumible para revestimiento duro E-83.58 (Tabla 1), que con la referencia del fabricante está indicado para revestimiento de piezas desgastadas como dientes de máquinas expuestas al desgaste en el sector minero.

Tabla 1: Composición química de los materiales ensayados (\% peso).

\begin{tabular}{lllllllll}
\hline MATERIALES & $\begin{array}{l}\text { COMPOSICIÓN } \\
\text { QUÍMICA }\end{array}$ & $\mathbf{C}$ & $\mathbf{M n}$ & $\mathbf{C r}$ & $\mathbf{N i}$ & $\mathbf{S i}$ & $\mathbf{M o}$ & $\mathbf{S}$ \\
\hline Oruga & Real & 0,36 & 0,98 & 0,17 & - & 0,22 & 0,13 & 0,02 \\
Postizo & Real & 0,67 & 0,66 & 0,23 & - & 0,10 & 0,15 & 0,02 \\
Cordón de & Nominal & 0,60 & 0,70 & 6,80 & - & 0,60 & 0,50 & - \\
Soldadura & Real & 0,79 & 0,79 & 10,68 & 0,15 & 0,78 & 0,49 & 0,02 \\
Electrodo E-83.58 & Nominal & 0,60 & 0,70 & 6,80 & - & 0,60 & 0,50 & - \\
Electrodo E-308-L & Nominal & 0,03 & 0,80 & 19,60 & 9,90 & 0,80 & - & - \\
\hline
\end{tabular}




\subsection{Metodología}

\subsubsection{Procedimiento de recuperación por soldadura}

Inicialmente fueron aplicadas capas intermedias (relleno) en seis pases longitudinales con electrodo E308-L, diámetro de 3,25 mm, con una composición química presentada en la Tabla 1, a continuación fue realizado un desbaste utilizando una lijadora rotativa hasta llegar a un espesor de la capa de 1,0 a 1,5 mm aprox.

La capa de relleno (dúctil) fue aplicada en el proceso SMAW para garantizar la buena unión entre el material base y el revestimiento duro, para evitar el agrietamiento inducidas por el Hidrogeno sobre el revestimiento, minimizar las tensiones y limitar el efecto de dilución (mudanza en la composición química de un metal de adición con el metal base).

El revestimiento duro consiste en cuatro capas de 4 pases longitudinales cada uno con un electrodo E-83.58, llegando a tener un espesor de $18 \mathrm{~mm}$ aproximadamente, seguido por el rectificado hasta reducir la probeta a $12,7 \mathrm{~mm}$ de espesura. La temperatura entre los pases fue controlada mediante el uso de termómetro digital de infrarrojo, manteniéndola inferior a $180^{\circ} \mathrm{C}$. La Figura 3 muestra esquemáticamente la aplicación de las capas del cordón de soldadura.

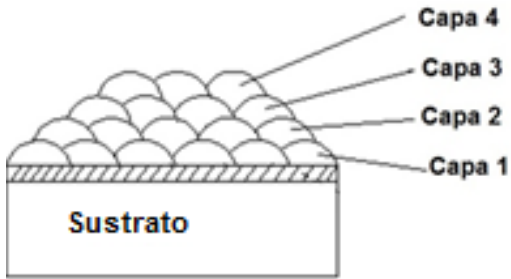

(a)

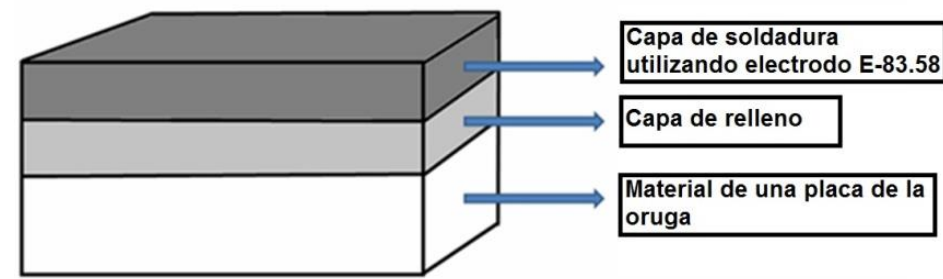

(b)

Figura 3: Representación esquemática de: (a) la aplicación de las capas, (b) sección transversal de la probeta con las capas de soldadura.

La Tabla 2 muestra los parámetros de soldadura utilizados en el proceso de deposición tipo SMAW. Los valores de corriente eléctrica se encuentran dentro de los límites establecidos por los fabricantes y la velocidad es compatible con las dimensiones de la pieza.

Tabla 2: Parámetros utilizados en el proceso de soldadura SMAW.

\begin{tabular}{lllll}
\hline $\begin{array}{l}\text { CÓDIGO DEL } \\
\text { ELECTRODO }\end{array}$ & $\begin{array}{l}\text { CORRIENTE } \\
(\mathbf{A})\end{array}$ & $\begin{array}{l}\text { VOLTAJE } \\
(\mathbf{V})\end{array}$ & $\begin{array}{l}\text { VELOCIDAD } \\
\left(\mathbf{m m} \cdot \mathbf{m i n}^{-1}\right)\end{array}$ & $\begin{array}{l}\text { APORTE DE CALOR } \\
(\mathbf{k J} / \mathbf{m m})\end{array}$ \\
\hline E-308-L & 100 & $26 \sim 29$ & 140 & $1,11 \sim 1,24$ \\
E-83.58 & 140 & $19 \sim 21$ & 137 & $0,83 \sim 0,92$ \\
\hline
\end{tabular}

\subsubsection{Caracterización de los materiales}

Las metalografías de los materiales fueron obtenidas después de pasar las probetas por la secuencia tradicional de lijado (\#220, \#330, \#400, \#600, \#800, \#1200 e \#1500), pulimiento con alúmina de 3,0 $\mu \mathrm{m}$ como abrasivo y ataque químico con solución de Nital al 2,0 \%.

El análisis de las metalografías se realizó utilizando imágenes de un microscopio óptico (MO) e para la observación de los mecanismos de desgaste se utilizó un microscopio electrónico de barrido (SEM).

Las medidas de dureza fueron obtenidas en escala Vickers con cargas de 100kgf para las probetas fabricadas de los materiales de la oruga y la soldadura y 30kgf para el postizo. La Figura 4, presenta los detalles de la medición en las probetas y en la garra de la oruga. 


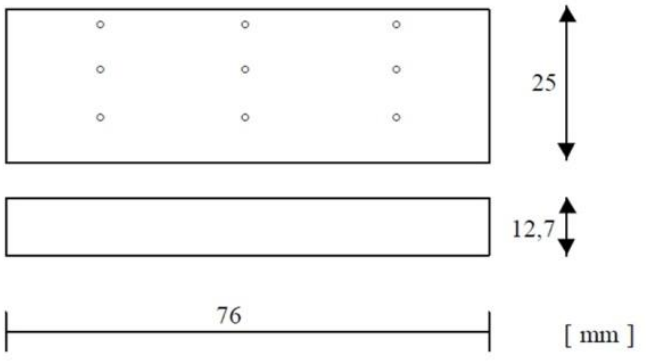

(a)

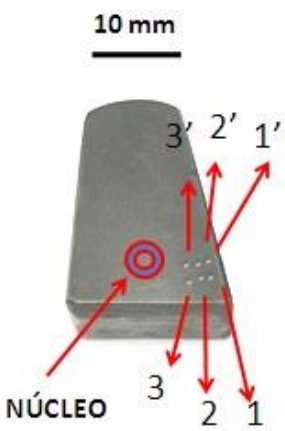

(b)

Figura 4: Detalle de la medición de dureza realizado en los diferentes materiales: (a) representación esquemática de las huellas del durómetro en las probetas de desgaste y (b) puntos de medición de dureza en la garra de la oruga.

\subsubsection{Ensayo de desgaste}

El ensayo de desgaste fue realizado mediante las especificaciones dadas por la norma ASTM G 65-04, con la máquina de rueda de caucho y chorro de arena seca, como es mostrado en la Figura 5.

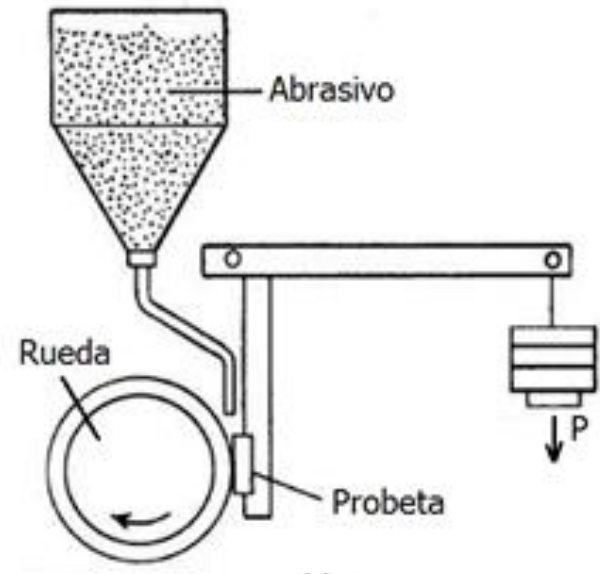

(a)

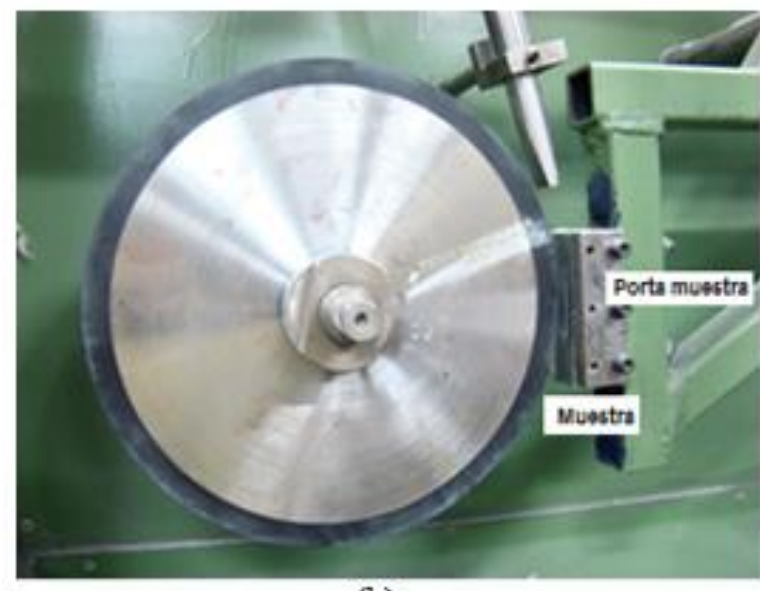

(b)

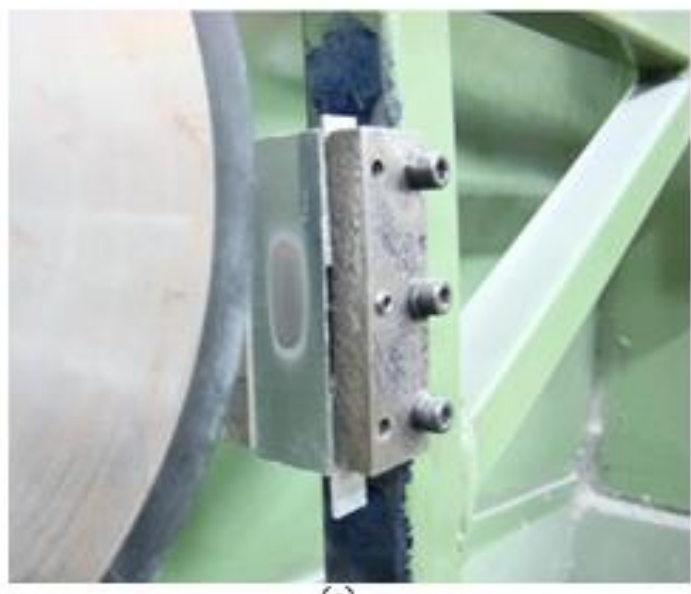

(c)

Figura 5: Detalles do equipo de desgaste: (a) Esquema general de la máquina, (b) posición de la muestra (probeta) en el equipo y (c) imagen de la huella de desgaste originada en el ensayo.

Las condiciones del ensayo fueron las mismas para cada uno de los diferentes materiales estudiados y son presentadas a continuación (Tabla 3). 
Tabla 3: Condiciones de ensayo de desgaste (método B de la norma ASTM G65-04).

\begin{tabular}{lll}
\hline CARACTERÍSTICAS & VALORES & UNIDADES \\
\hline Dimensiones da las muestras & $76,2 \times 25,4 \times 12,7$ & $\mathrm{~mm}$ \\
Diámetro del disco & 228 & $\mathrm{~mm}$ \\
Carga aplicada & 130 & $\mathrm{~N}$ \\
Rotación del disco & 200 & $\mathrm{rpm}$ \\
Dureza del caucho & 60 & Shore A \\
Distancia recorrida del ensayo & 1436 & $\mathrm{~m}$ \\
Flujo de alimentación de arena & $175,1 \pm 11,5$ & $\mathrm{~g} \cdot \mathrm{min}^{-1}$ \\
\hline
\end{tabular}

El abrasivo utilizado fue la arena normal brasileira, granulometría 100 (tamaño promedio de 0,15 mm y dureza de $~ 1100 \mathrm{HV}$ ) según la norma ABNT NBR 7214:2012 [22]

Debido al desgaste ocurrido en la rueda por la sucesión de ensayos, fue realizada la corrección en los valores de pérdida de masa considerando el diámetro inicial y final de la rueda, esta corrección es comúnmente llamada de pérdida de masa corregida $(P M C)$. Considerando esta mudanza en el sistema tribológico, la Norma ASTM G65-04 propone el uso de la ecuación 1 a continuación,

$$
P M C[g]=\left(m_{i}-m_{f}\right) \cdot \frac{\phi_{i}}{\phi_{f}} \quad x 1000
$$

En la cual:

- $\quad m_{i}:$ a masa inicial de las muestras (probetas);

- $\quad m_{f}$ : a masa final de las muestras.

El diámetro inicial $\left(\phi_{i}\right)$ fue considerado según referencia de la norma (228 $\left.\mathrm{mm}\right)$ y el diámetro final $\left(\phi_{f}\right)$ fue medido después de cada ensayo, convirtiéndose este en el nuevo diámetro inicial para el ensayo siguiente.

Para facilitar la comparación de las pérdidas de masa de los diferentes materiales, es necesario convertir la pérdida de masa en pérdida de volumen corregida $(P V C)$ y encontrar la resistencia al desgaste $\left(Q^{\prime}\right)$, que es el inverso del cociente de la perdida de volumen por la distancia recorrida durante el ensayo como es presentado en las ecuaciones (2) y (3).

$$
P V C\left[\mathrm{~mm}^{3}\right]=\frac{P M C}{\text { masa específica }}=\frac{\left(m_{i}-m_{f}\right) \cdot\left(\phi_{i} / \phi_{f}\right)}{\delta} \times 1000
$$

En la cual:

$$
\begin{aligned}
&- \delta: \text { la masa específica } \\
& \bullet \quad \text { oruga, 7,84 g.cm } \mathrm{cm}^{-3} \\
& \bullet \quad \text { postizo } 7,87 \mathrm{~g} \cdot \mathrm{cm}^{-3} \\
& \text { cordón de soldadura: } 7,87 \mathrm{~g} \cdot \mathrm{cm}^{-3} \\
& Q^{\prime}\left[\frac{m}{\mathrm{~mm}^{3}}\right]=\left(\frac{P V C}{\text { dist. }}\right)^{-1}
\end{aligned}
$$

Para hacer una comparación de comportamiento con los resultados de otros autores [23,$\underline{24}]$, también puede definir la resistencia másica al desgaste $\left(Q^{\prime \prime}\right)$ como la ecuación (4).

$$
Q^{\prime \prime}\left[\frac{m}{m g}\right]=\left(\frac{P M C}{\text { dist. }} x 1000\right)^{-1}
$$




\section{RESULTADOS Y DISCUSIONES}

\section{Análisis microestructural}

En la Figura 6 se pueden apreciar las micrografías del postizo y la oruga cerca de la superficie de desgaste.

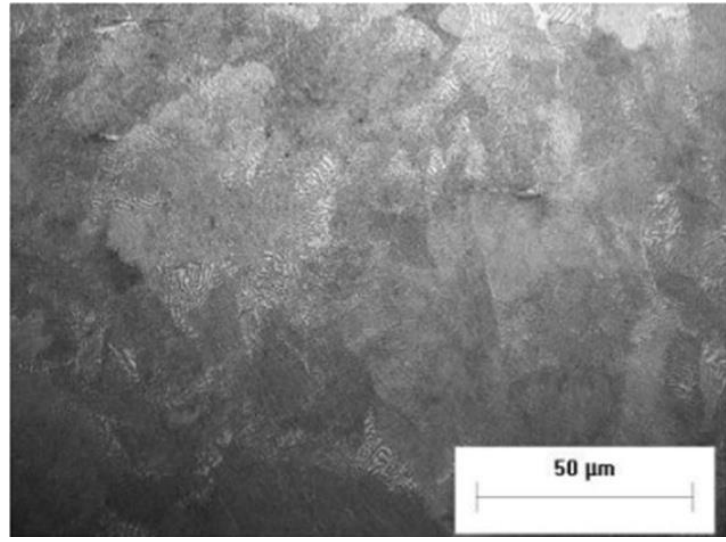

(a)

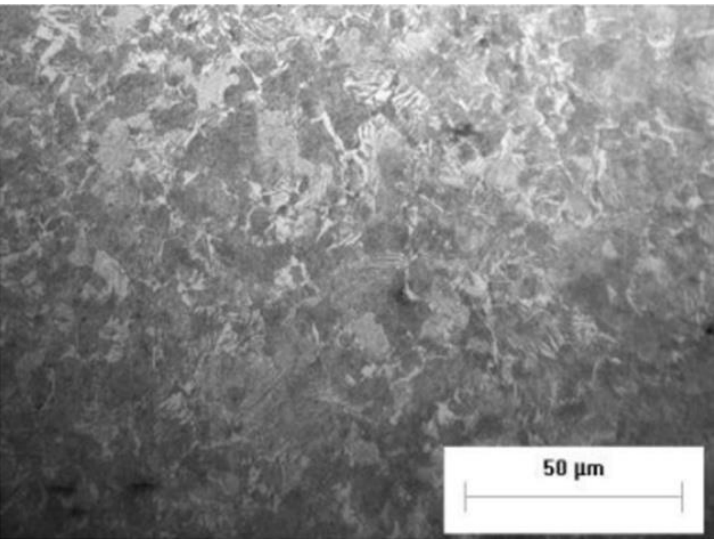

(b)

Figura 6: Micrografías con amplificación de 500x y ataque de Nital 2\%: (a) material del postizo, (b) material de la oruga.

En la Figura 6 se observa o predominio de una matriz perlítica con presencia de pocas áreas de ferrita, con una refinamiento de grano mayor para o material de la oruga. En oruga es posible observar una diferencia microestructural significativa de la superficie con respecto al núcleo (destacando el tamaño de grano), con una capa de aproximadamente $35 \mu \mathrm{m}$. Este hecho está de acuerdo con el proceso de fabricación de la oruga que es laminación seguido por temple y revenido. soldadura.

En la Figura 7 se pueden apreciar las tres diferentes zonas presentes en la probeta con cordón de

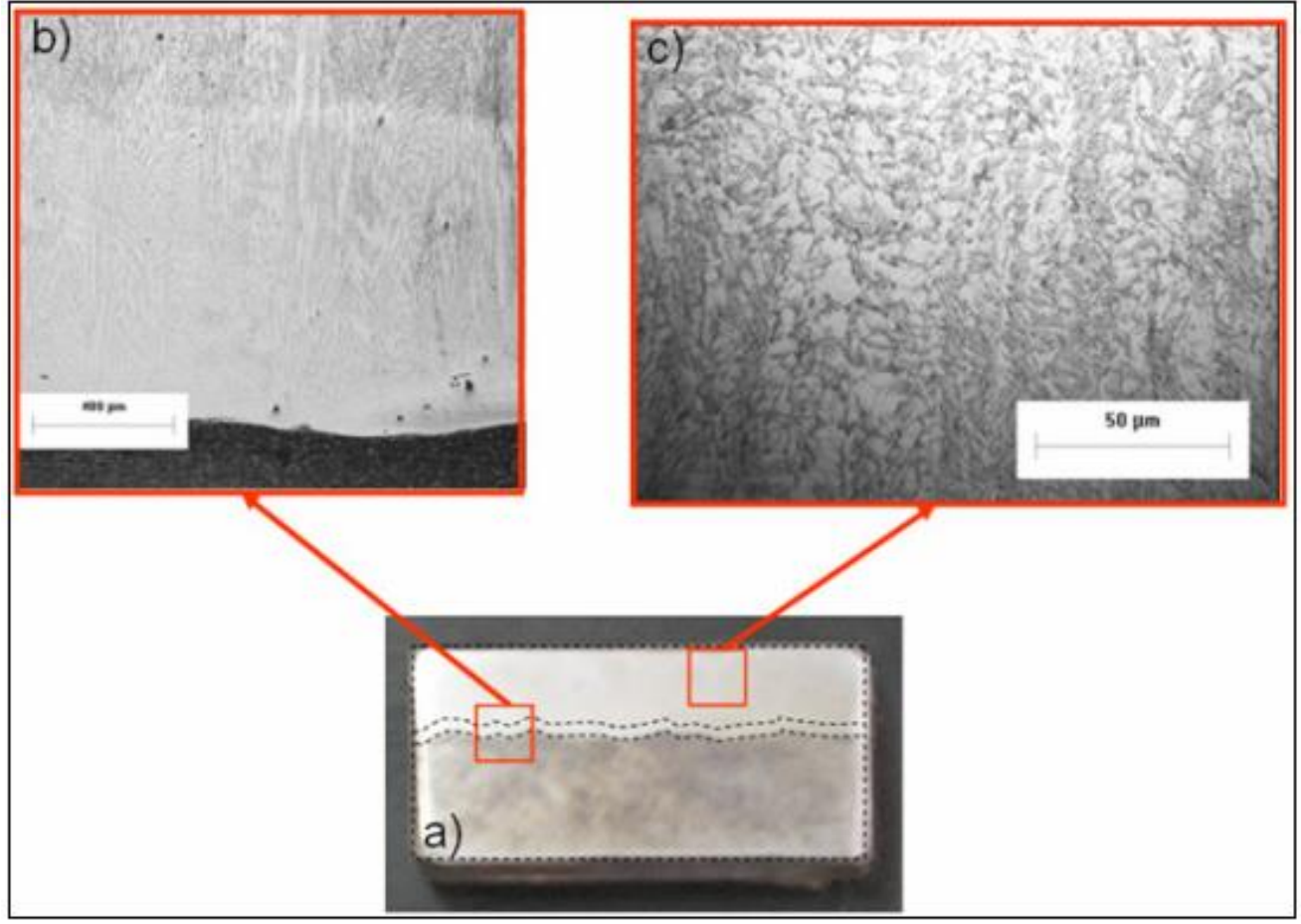

Figura 7: Micrografías del cordón de soldadura: (a) macrografía con identificación de tres zonas distintas, (b) interface: relleno y soldadura y (c) región de la triple capa de soldadura. 


\section{Análisis de dureza}

Los resultados promedios del análisis de dureza de los diferentes materiales está siendo presentado en la Tabla 4.

Tabla 4: Valores de dureza Vickers de los diferentes materiales

\begin{tabular}{lll}
\hline POSTIZO & ORUGA & SOLDADURA \\
\hline $270 \pm 6$ & $447 \pm 22$ & $490 \pm 40$ \\
\hline
\end{tabular}

La caracterización metalográfica presentada en la Figura 7 muestra la presencia de porosidad y defectos provenientes del proceso de soldadura que tienen clara influencia en los valores de dureza y la dispersión encontrada.

Analizando la dureza del postizo se verifica que ésta es compatible con un acero SAE/AISI 1060, tanto por la composición como por el aporte térmico recibido al momento de la unión con el material de la oruga (proceso de unión por soldadura a arco sumergido con enfriamiento lento), lo que acabo por promover una normalización de este material y consecuentemente llevando a una dureza menor que la del material de la oruga, presentada a continuación en la Tabla 5.

Tabla 5: Perfil de dureza del material de la garra de la oruga.

\begin{tabular}{llll}
\hline POSICIÓN & $\begin{array}{l}\text { DUREZA } \\
\text { (HV) }\end{array}$ & POSICIÓN & $\begin{array}{l}\text { DUREZA } \\
\text { (HV) }\end{array}$ \\
\hline 1 & 434 & 1 & 446 \\
2 & 402 & 2 & 434 \\
3 & 354 & 3 & 382 \\
\hline
\end{tabular}

Analizando los valores de dureza Vickers del material de la oruga, mostrados en la Tabla 5, en la región de la garra se ha observado que esta no es uniforme, puesto que se encuentran durezas diferentes en la región del núcleo (Figura 4b) de 368 HV con la región de la superficie de la garra próxima a 440 HV. Este hecho puede ser considerado aceptable, pues la oruga en sus últimas etapas de fabricación fue laminada, templada y revenida.

En el análisis del cordón de soldadura se pueden encontrar 3 diferentes zonas de dureza observados en las impresiones dejadas por el equipo de análisis. Esta información puede verse en la Figura 8, siendo observable el incremento gradual en la región de relleno, obtenido posiblemente por la dilución de los materiales del electrodo de alta dureza y del electrodo utilizado para el relleno (E-308-L).

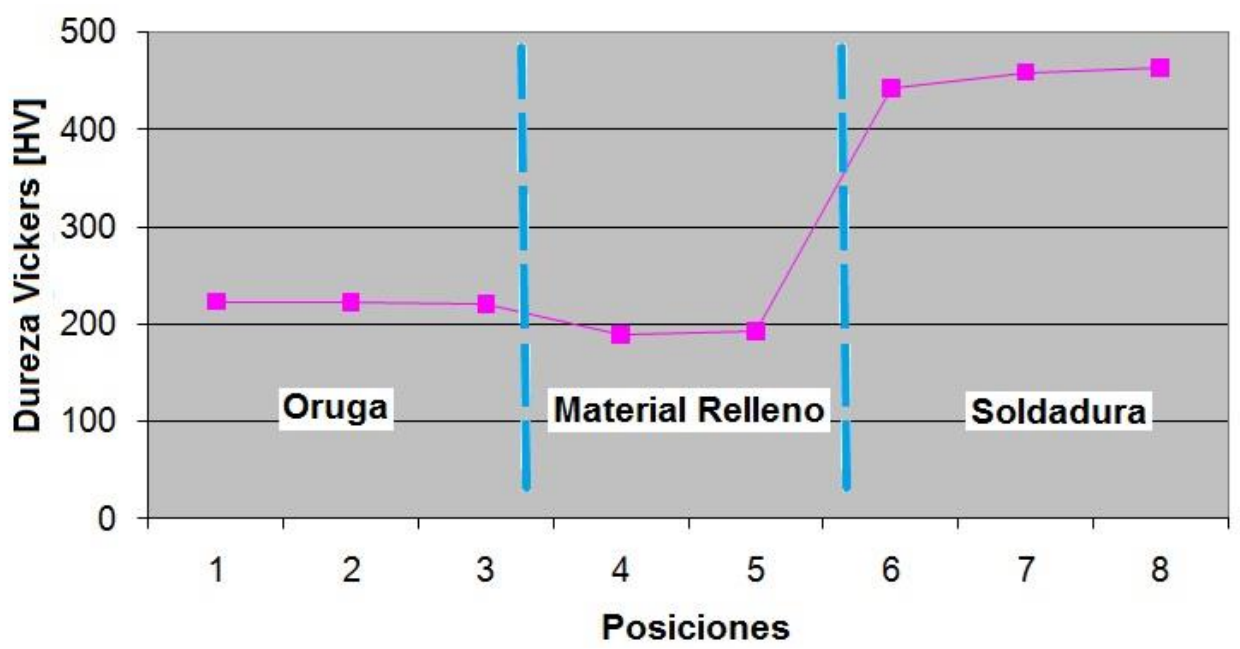

Figura 8: Perfil de dureza Vickers de la muestra con deposición de los cordones de soldadura. 


\section{Grietas Superficiales}

Analizando el revestimiento duro utilizado en este trabajo, con un tenor de elementos de liga del $20 \%$, más específicamente con $0,79 \%$ C; $0,78 \% \mathrm{Si} ; 0,79 \% \mathrm{Mn} ; 10,68 \%$ Cr e $0,49 \%$ Mo, se nota que este presento valores de dureza aproximada de $490 \mathrm{HV}$, resultado mayor al obtenido por la oruga $(447 \pm 22$ $\mathrm{HV}$ ). Las condiciones de soldadura, principalmente la temperatura entre los pases (manteniendo inferior a $180^{\circ} \mathrm{C}$ ) y el aumento de la dureza no provocan el surgimiento de grietas significativas cuando se compara con los resultados de otros investigadores, conforme se ha presentado en la Figura 9.

RIBEIRO [23] utilizó un electrodo revestido con composición química 5,3\% C; 1,2\% Si; 0,8\% Mn e $42,0 \% \mathrm{Cr}$, con una dureza aproximada de $784 \mathrm{HV}$, sin embargo, en sus conclusiones verifico que pocas muestras no presentaron grietas en la superficie del revestimiento. La Figura 9c muestra una probeta con diversos defectos debido a la soldadura y una visible grieta transversal. ARNT et al. [24] utilizó un electrodo con composición química de 4,8\% C; $0,93 \%$ Si; $0,16 \%$ Mn; $18,9 \%$ Cr; 6,82\% Nb y 0,4\% B. La dureza encontrada fue de $746 \mathrm{HV}$ y también se constató la presencia de grietas conforme la Figura 9d.

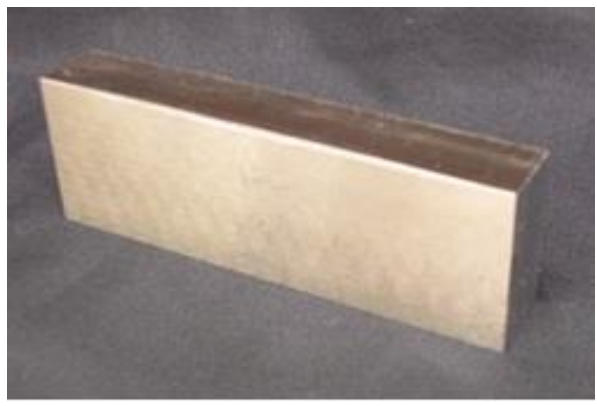

(a)

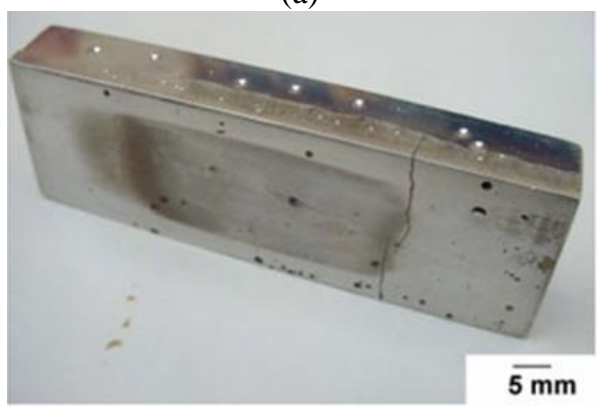

(c)

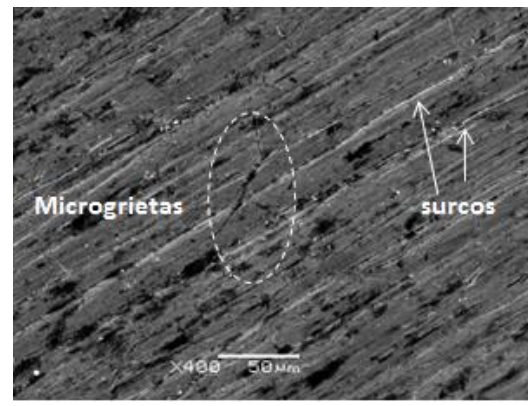

(b)

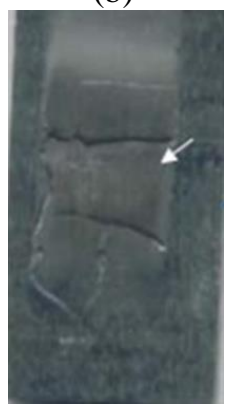

(d)

Figura 9: Imágenes para análisis de grietas superficiales: (a) Fotografía de una muestra con soldadura antes de los ensayos de desgaste, (b) imagen SEM microgrietas y riscos en la región de ensayos de desgaste, (c) imagen de muestra obtenida de la investigación de RIBEIRO [23] y (d) imagen de muestra obtenida de la investigación de ARNT et al. [24].

Según LIMA y FERRARESI [25] es recomendable que al elegir una aleación para el revestimiento duro se debe llevar en cuenta de su soldabilidad, costos y la compatibilidad metalúrgica. Las aleaciones con alto tenor de $\mathrm{Fe}-\mathrm{Cr}-\mathrm{C}$ son particularmente atractivas por que los carburos resultantes pueden formar una gran variedad de micro-constituyentes generando un aumento de resistencia a la abrasión. Sin embargo, estas aleaciones son susceptibles a las grietas de solidificación, las cuales alivian las tensiones de soldaduras, pero en casos en los cuales los componentes están sujetos a vibración o impacto pueden llevar a fragmentación del revestimiento.

\section{Resistencia y mecanismos de desgaste}

La Figura 10 presenta la relación de pérdida de masa corregida con dureza de los materiales, mostrando que aun siendo mayor la dureza de la oruga original cuando se compara con el postizo presenta un mayor nivel de desgaste, correspondiendo esta pérdida de masa a $12 \%$ superior. Cuando se comparado con el proceso de soldadura (siendo este el de mejor respuesta tribológica), el material original perdió $25 \%$ más de masa. 


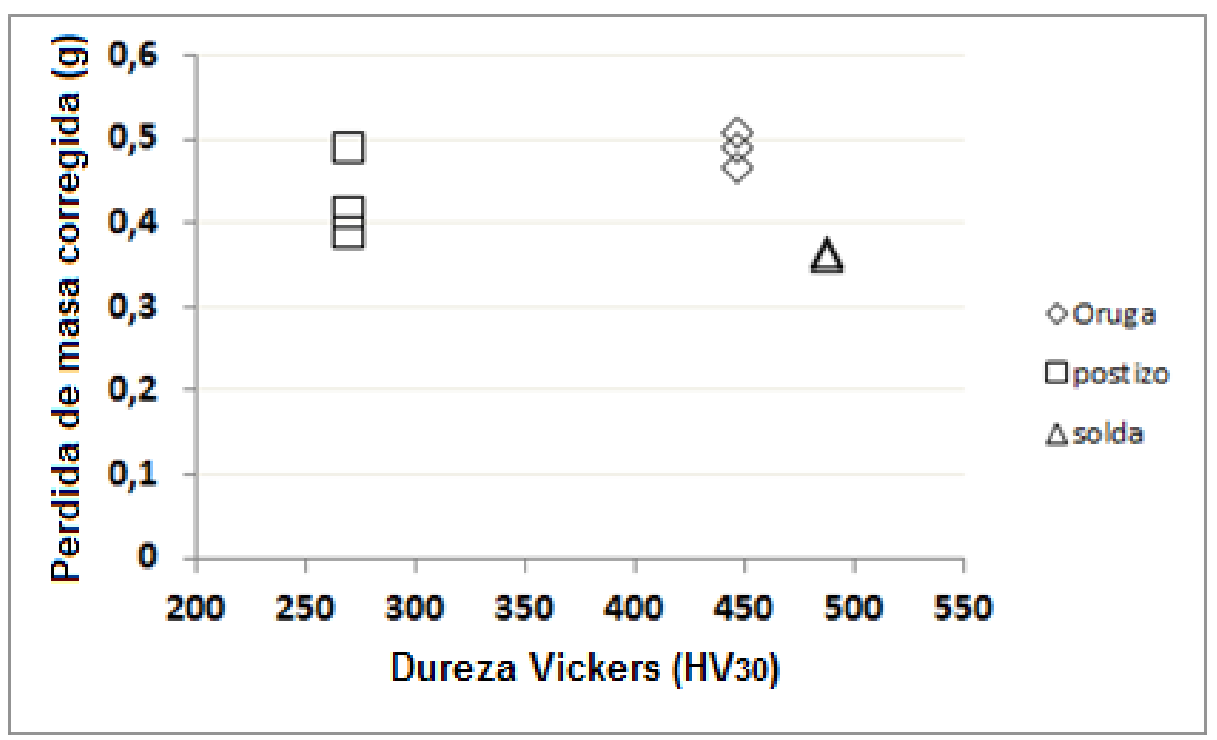

Figura 10: Pérdida de masa en función de la dureza de los materiales.

Los resultados presentados en la Figura 10 muestran que la dureza de la oruga es superior cuando se compara con la dureza del postizo, pero tiene una mayor pérdida de masa. Este resultado puede ser explicado basados en los resultados de RIBEIRO [23] y de KOTECKI y OGBORN [24], quien concluyó que la dureza no es el mejor parámetro a utilizar para analizar la resistencia al desgaste abrasivo y si la microestructura del material.

Otro factor de importancia es la disminución de la dureza en la medida que se aproxima al núcleo. La Figura 11 muestra la región de la superficie de la muestra soldada, indicando una profundidad de desgaste de $430 \mu \mathrm{m}$. Si se considera que la capa endurecida de la oruga no es muy elevada, se puede concluir que la capa superficial en donde se observa una dureza mayor es rápidamente eliminada por el proceso de desgaste, tanto no ensayo tribológicos como en el trabajo en campo.

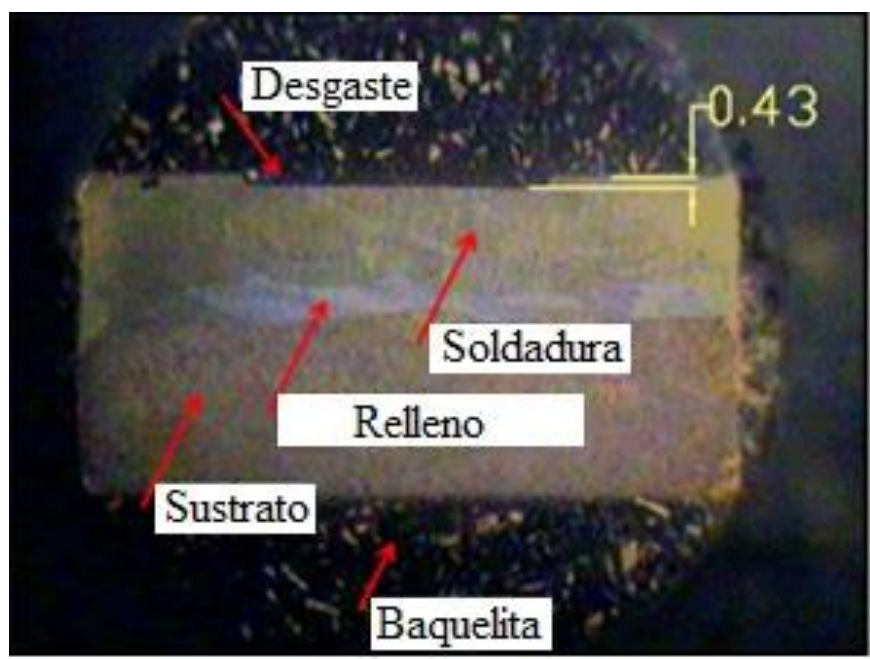

Figura 11: Fotografía de la sección transversal de la muestra indicando la profundidad del desgaste (mm) y las diferentes regiones presentes: sustrato, relleno e soldadura.

La Tabla 6 presenta los resultados generales de los materiales analizados en este estudio, mostrándolos según norma ASTM en pérdida de masa y de volumen con las correcciones necesarias debido al desgaste de la rueda de caucho. 
Tabla 6: Resultado general de los ensayos de desgaste según ASTM G65-04.

\begin{tabular}{lccc}
\cline { 2 - 3 } & ORUGA & POSTIZO & SOLDADURA \\
\hline $\begin{array}{l}\text { Pérdida de masa corregida } \\
P M C(\mathrm{~g})\end{array}$ & $0,49 \pm 0,02$ & $0,40 \pm 0,01$ & $0,363 \pm 0,004$ \\
$\begin{array}{l}\text { Pérdida de volumen corregida } \\
P V C\left(\mathrm{~mm}^{3}\right)\end{array}$ & $59 \pm 2$ & $48 \pm 1$ & $43,7 \pm 0,5$ \\
$\begin{array}{l}\text { Resistencia al desgaste } \\
Q^{\prime}\left(\mathrm{mm}^{3} \cdot \mathrm{m}^{-1}\right)^{-1}\end{array}$ & $23,0 \pm 1,1$ & $28,3 \pm 0,9$ & $31,1 \pm 0,4$ \\
$\begin{array}{l}\text { Resistencia másica al desgaste } \\
Q^{\prime \prime}\left(\mathrm{mg} \cdot \mathrm{m}^{-1}\right)^{-1}\end{array}$ & $3,1 \pm 0,1$ & $3,8 \pm 0,1$ & $4,2 \pm 0,1$ \\
\hline
\end{tabular}

BUCHELY et al. [17] en un estudio similar con deposición de soldadura en multicapas presenta resultados comparable, no obstante, tanto el proceso de soldadura como las aleaciones depositadas (todas ricas en Cromo, Tungsteno y Molibdeno, además de Niobio y Vanadio) presentan costos operacionales más altos que los utilizados en este trabajo.

Por otra parte, resultados semejantes fueron registrados por LIMA y FERRARESI [26], quienes utilizaron el proceso FCAW (electrodo tubular con alto contenido de cromo) y el proceso SMAW (electrodo revestido), mostrando que la utilización de procesos de soldadura presentan resultados superiores de resistencia al desgaste (relación de pérdida de masa) que la utilización de postizos.

La Figura 12 muestra imágenes de la superficie de desgaste de las muestras obtenidas en las garras de la oruga después de sufrir desgaste por trabajo en el campo. Puede observarse presencia de deformación plástica (con microsurcos) además de grietas (característica del mecanismo de corte).

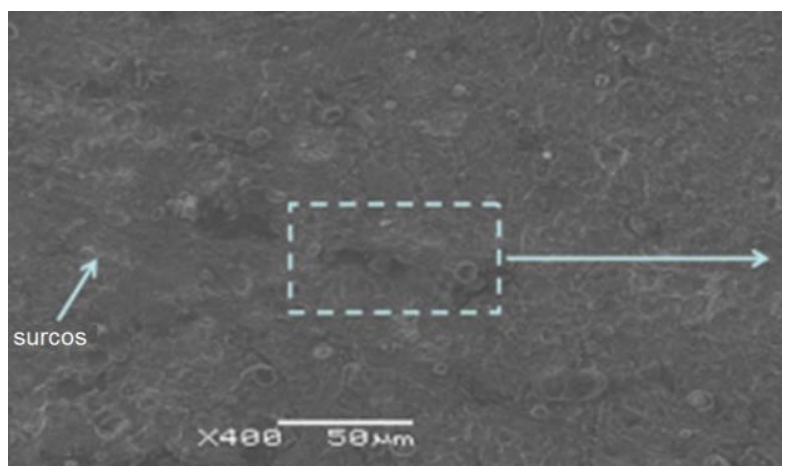

(a)

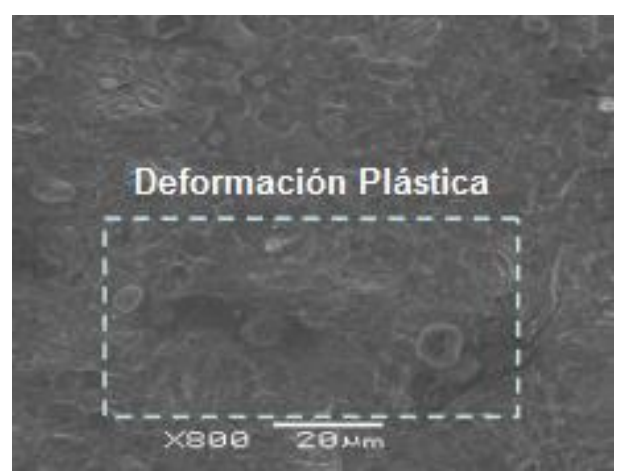

(b)

Figura 12: Imagen SEM de la superficie de la garra de la oruga desgastada en el campo presentando: (a) microsurcos y deformación plástica, (b) detalles de la zona con deformación plástica.

La dureza del abrasivo influye en el grado de penetración en la superficie del material, por lo tanto, influencia también en la tasa de desgaste. La Tabla 7 presenta los valores de la razón de dureza $\mathrm{H}_{\mathrm{a}} / \mathrm{H}$ de cada material estudiado.

Tabla 7: Razón de dureza $\mathrm{H}_{\mathrm{a}} / \mathrm{H}$ de los materiales.

\begin{tabular}{l|lll}
\hline MEDICIONES & POSTIZO & ORUGA & SOLDADURA \\
\hline Dureza Promedio & 270 & 447 & 490 \\
$\mathbf{H}_{\mathrm{a}} / \mathbf{H}$ & 4,07 & 2,46 & 2,24 \\
\hline
\end{tabular}

PEREIRA [10] muestra que la relación $\mathrm{H}_{\mathrm{a}} / \mathrm{H}$ apesar de ser una forma importante de clasificar el régimen de desgaste para sistemas bajo condiciones similares de dureza de los abrasivos, las características de las partículas abrasivas (distribución de tamaños y forma) se muestra relevante a la hora de analizar los resultados de tasa de desgaste. Por lo tanto, para sistemas con postizo y oruga, no existe 
una relación directa entre $\mathrm{H}_{\mathrm{a}} / \mathrm{H}$ y resistencia al desgaste.

La Tabla 8 muestra de forma detallada el comportamiento de cada uno de los materiales en estudio, observándose los mecanismos de desgaste predominantes influenciados por deformación plástica.

Tabla 8: Relación de resultados obtenidos en los diferentes componentes.

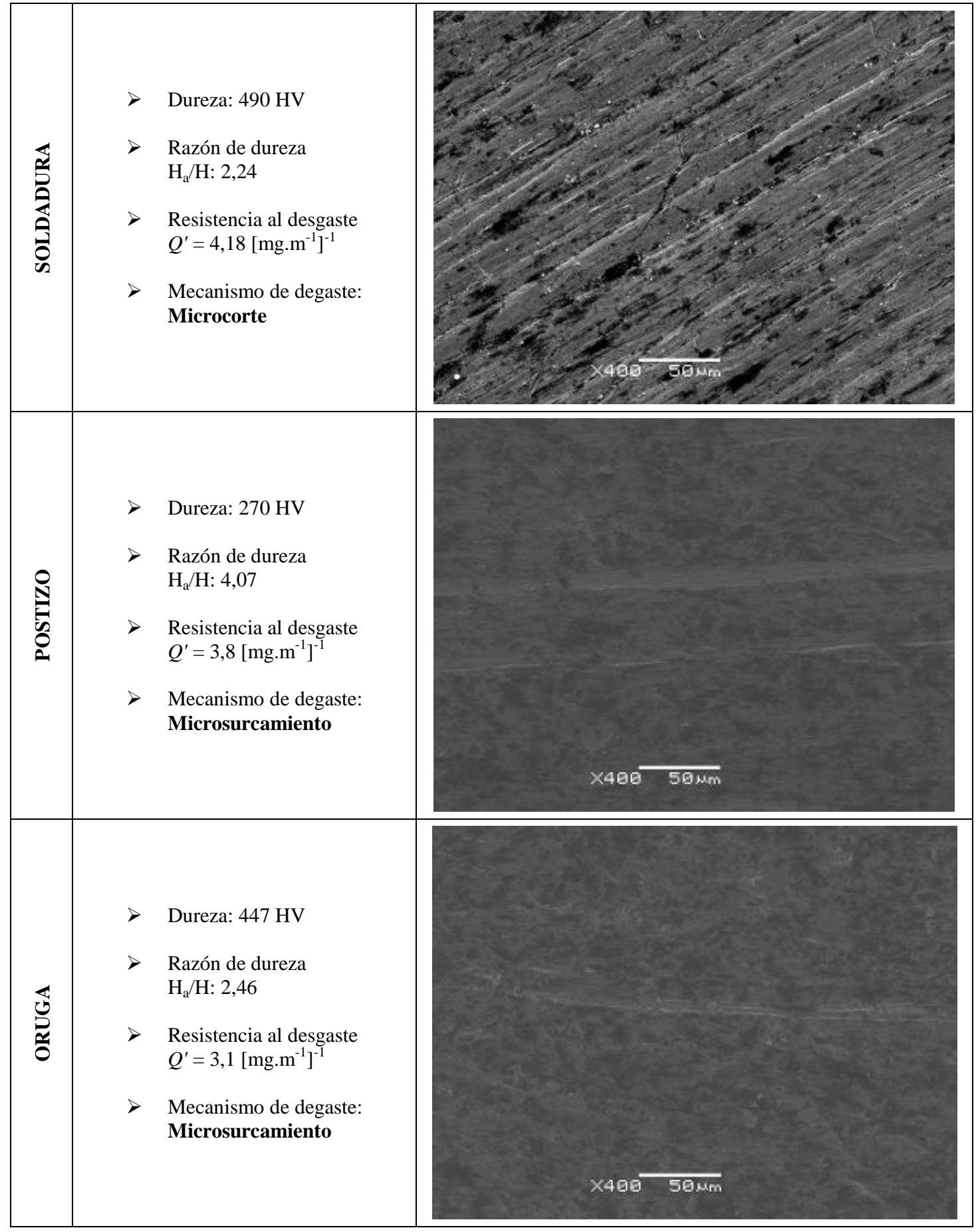

Según LIMA y FERRARESI [26] la dureza del abrasivo influye en el grado de penetración en la superficie del material, por lo tanto, también en la tasa de desgaste. PEREIRA [10] muestra que la razón $\mathrm{H}_{\mathrm{a}} / \mathrm{H}$ a pesar de ser una forma importante de clasificar el régimen de desgaste para sistemas bajo condiciones similares de dureza de los abrasivos, las características de las partículas abrasivas (distribución de tamaños y forma) se muestra relevante a la hora de analizar los resultados de tasa de desgaste. Por lo tanto, para sistemas con postizo y oruga, no existe una relación directa entre $\mathrm{H}_{\mathrm{a}} / \mathrm{H}$ y 
resistencia al desgaste.

Con el análisis de los resultados mostrados en la Tabla 8, es posible obtener las siguientes conclusiones en relación a los micromecanismos de desgaste:

En la oruga y el postizo, se observa poca presencia de micromecanismo de corte, siendo predominante entonces la presencia de surcamiento, y mostrando que no existe diferencia significativa entre los dos materiales;

La resistencia al desgaste del postizo es superior a la de la oruga un teniendo esta ultima una mayor dureza (mayor resistencia mecánica);

La muestra de soldadura es aquella que presenta mayor resistencia mecánica e resistencia al desgaste, siendo observado sin embargo la presencia de corte como micromecanismo de desgaste predominante.

En términos generales puede observarse la presencia de corte en las muestras de soldadura (aquella que presenta mayor dureza) en contrapartida de las muestras de las otras muestras en las cuales se evidencia deformación plástica (surcos) con o sin formación de proa, resultado que puede relacionarse con los obtenidos por HOKKIRIGAWA et al. [27]. El grupo investigador mostro que es posible pasar de mecanismo de microsurcado para microcorte con el aumento de la dureza del material de la superficie para un ángulo de ataque similar y relaciona la eficiencia de corte de las partículas abrasivas al aumento de la dureza superficie de desgaste.

\section{CONCLUSIONES}

Los resultados obtenidos en este trabajo permitieron obtener las siguientes conclusiones: La soldadura con aleación inferior de $20 \%$ presento dureza compatible con el material a ser recuperado. La soldadura presento una mejor resistencia al desgaste, principalmente cuando se compara con el postizo o la oruga original. La superficie de la soldadura presento buena apariencia al ser comparada con las superficies de los materiales con mayor dureza. Se obtiene microcorte como el micromecanismo de desgaste predominante en la soldadura no siendo posible evidenciar la presencia de deformación plástica obedeciendo a su elevada dureza y baja capacidad de deformación. Por otro lado para la oruga y el postizo (ambos aceros con una conocida capacidad de deformación) presentaron deformación plástica con predominio de microsurcamento. La recuperación por soldadura y/o deposición de capas de electrodo para aplicación en campo, mostro ser una solución bastante adecuada para el mantenimiento de tractores tipo Bulldozer.

\section{AGRADECIMIENTOS}

Los autores agradecen el apoyo de las siguientes instituciones: IFAM - Instituto Federal de Educação, Ciência e Tecnologia do Amazonas; Laboratório de Superfícies e Contato (LASC-DAMEC); Programa de Pós-Graduação em Engenharia Mecânica e de Materiais (PPGEM-UTFPR), Fundação de Apoio à Pesquisa do Estado do Amazonas (FAPEAM) y Superintendência da Zona Franca de Manaus (SUFRAMA).

\section{BIBLIOGRAFÍA}

[1] VILLABÓN, L.R., SINATORA, A., "Construção e instrumentação de abrasômetro do tipo roda-deborracha para o estudo do comportamento tribológico de aços", APAET - Mecânica Experimental, v.13, pp. 1-11, 2006.

[2] CZICHOS, H., HABIG, K.H., Tribologie-Handbuch, Friedr. Vieweg \& Sohn, 2 Ed., 2003.

[3] ZUM GAHR, K.-H., "Microstructure and wear of materials", Elsevier, The Netherlands, 1987.

[4] ASTM G 40-01, American Standard for Testing Materials. Standard Terminology Relating to Wear and Erosion. Annual Book of Standards Volume 03.02: Wear and Erosion; Metal Corrosion. Philadelphia, USA, 2001.

[5] RABINOWICZ, E., DUNN, L. A., RUSSEL, P.G., "A study of abrasive wear under three-body conditions", Wear, v. 4, pp. 345 - 355, 1961.

[6] GATES, J.D., "Two-body and three body abrasion: A critical discussion”, Wear, v.214, pp. 139-146, 1998. 
[7] ASTM G 65-04, American Standard for Testing Materials. Measuring abrasion using the dry sand/rubber wheel apparatus, Annual Book of Standards Volume 03.02: Wear and Erosion; Metal Corrosion. Philadelphia, USA, 2001.

[8] HUTCHINGS, I.M., "Development of the dry sand/rubber wheel abrasion test", Wear, v. 195, pp. 232-240, 1996.

[9] NAHVI, S.M., "Effects of particle crushing in abrasion testing of steels with ash from biomass-fired powerplants", Wear, v. 267, pp.34-42, 2009.

[10] PEREIRA, J.I., Efeito da forma da partícula abrasiva no desgaste de ligas de alumínio e de aço usadas na fabricação de cavidades para moldes de injeção de termoplásticos, Dissertaçao de Mestrado, Universidade Tecnológica Federal do Paraná, Curitiba, PR, Brasil, 2011.

[11] RUTHERFORD, K. L., HUTCHINGS, I. M., "A micro-abrasive wear test, with particular application to coated systems", Surface and Coatings Technology, v. 79, n. 1-3, pp. 231-239, 1996.

[12] FANG, L., KONG, X. L., SU, J. Y., et al., "Movement patterns of abrasive particle in three-body abrasion", Wear, v. 162-164, pp. 782-789, 1993.

[13] HUTCHINGS, I.M., Tribology: Friction and wear of Engineering Materials, Edward Arnold, London, 1992.

[14] KHRUSCHOV, M.M., "Principles of abrasive wear", Wear, v. 28, pp. 69-88, 1974.

[15] MONTEIRO, M. P., CUNHA, J. A. R., “Tecnologia de arames tubulares com Proteção gasosa para revestimento anti-desgaste para aplicações em usinas sucroalcooleiras e mineradoras", In: Anais do $19^{\circ}$ Encontro Nacional de Tecnologia da Soldagem, Águas de São Pedro, pp. 195-210, São Paulo, 1993.

[16] BRANDIM, A. S., ALCÂNTARA, N. G., HERNANDEZ, O. J. S., et al., Resistência ao Desgaste por abrasão em metal de solda do tipo $\mathrm{Fe}-\mathrm{C}-\mathrm{Cr}$, obtidos por arco submerso, In: $2^{\circ}$ Congresso Brasileiro de Engenharia de Fabricação, Uberlândia, 2003

[17] BUCHELY, M.F., GUTIERREZ, J, C., LEON, L.M., "The effect of microstructure on abrasive wear hardfacing alloys", Wear, v. 259, pp. 52-61, 2005.

[18] FERNANDEZ, J.E., VIJANDE, R., TUCHO, R., "Effect of cold deformation on the abrasive resistance of coatings with applications in mining industry", Wear, v. 250, pp. 28-31, 2001.

[19] RICARDO, H. S., CATALANI, G., Manual prático de escavação - Terraplenagem e escavação de rocha, Pini, São Paulo, 1990.

[20] KOMATSU Manual técnico, http://www.komatsueurope.com , Accesado en Febrero de 2012.

[21] SOTREC. Manual de Operação e Manutenção, Caterpillar, São Paulo, 2000.

[22] ABNT NBR 7214:2012, Areia normal para ensaio de cimento. Associação Brasileira de Normas Técnicas, 2012.

[23] RIBEIRO, R., Avaliação da resistência ao desgaste abrasivo de revestimentos soldados do tipo Fe-CCr utilizados na indústria sucroalcooleira, Dissertação (Mestrado), Faculdade de Engenharia de Ilha Solteira, Universidade Estadual Paulista, São Paulo, SP, Brasil, 2004.

[24] ARNT, A. B. C., ROCHA, M. R., TORRES, L. A., Avaliação do desempenho de revestimentos depositados por solda em rolos de moagem de carvão, In: $17^{\circ}$ Congresso Brasileiro de Engenharia e Ciência dos Materiais, 2006.

[25] KOTECKI, D.J., OGBORN, J.S., “Abrasion resistance of iron-based hardfacing alloys", Welding Research Supplement, pp. 269-278, Aug. 1995.

[26] LIMA, A. C., FERRARESI, V. A., “Análise da Microestrutura e da Resistência ao Desgaste de Revestimento Duro Utilizado pela Indústria Sucroalcooleira”, Soldagem \& Inspeção, v. 14, n. 2, pp. 140$150,2009$.

[27] HOKKIRIGAWA, K., KATO, K., LI, Z.Z., "Effect of hardness on the transition of the abrasive wear mechanism of steels", Wear, v.123, pp. 241-251, 1987. 